University of Nebraska - Lincoln

DigitalCommons@University of Nebraska - Lincoln

$6-1-2004$

\title{
Multidomain and incoherent effects in magnetic nanodots
}

\author{
Ralph Skomski \\ University of Nebraska-Lincoln, rskomski2@unl.edu \\ Arti Kashyap \\ University of Nebraska-Lincoln, akashyap@Inmiit.ac.in \\ Kory D. Sorge \\ University of Nebraska-Lincoln, sorge@physics.fau.edu \\ David J. Sellmyer \\ University of Nebraska-Lincoln, dsellmyer@unl.edu
}

Follow this and additional works at: https://digitalcommons.unl.edu/physicssellmyer

Part of the Physics Commons

Skomski, Ralph; Kashyap, Arti; Sorge, Kory D.; and Sellmyer, David J., "Multidomain and incoherent effects in magnetic nanodots" (2004). David Sellmyer Publications. 24.

https://digitalcommons.unl.edu/physicssellmyer/24

This Article is brought to you for free and open access by the Research Papers in Physics and Astronomy at DigitalCommons@University of Nebraska - Lincoln. It has been accepted for inclusion in David Sellmyer Publications by an authorized administrator of DigitalCommons@University of Nebraska - Lincoln. 


\title{
Multidomain and incoherent effects in magnetic nanodots
}

\author{
R. Skomski, ${ }^{\text {a) }}$ A. Kashyap, K. D. Sorge, and D. J. Sellmyer \\ Department of Physics and Astronomy and Center for Materials Research and Analysis, \\ University of Nebraska, Lincoln, Nebraska 68588
}

(Presented on 8 January 2004)

\begin{abstract}
The magnetic ground state and the magnetization reversal of aspherical nanoparticles are investigated by model calculations and micromagnetic simulations. Essential deviations from Kittel's domain theory occur for very flat and very elongated particles, for particles whose size is comparable to the domain-wall width, and when the particle shape is strongly nonellipsoidal. For example, the single-domain state of square rod is much less stable than that of comparable elongated ellipsoids, because most of the surface charge of long ellipsoids is located on the sides. Another specific feature of flat particles is suppression of the curling mode. (C) 2004 American Institute of Physics. [DOI: 10.1063/1.1688645]
\end{abstract}

\section{INTRODUCTION}

Interest in magnetic nanoparticles has been fueled by numerous present and future applications in advanced technology, from ferrofluids and particulate magnetic recording media to quantum computing. Historically, small magnetic particles were first investigated in the mid 20th century, 1,2 and concept such as Stoner-Wohlfarth particles originate from that time. The continuing interest in these particles has two aspects: new structures and new geometries. For several decades, nanoparticles where made from soft or semihard materials and had more or less elongated shapes. Now, the thickness, width, length, and geometry of nanoparticles can be varied over a wide range, ${ }^{3,4}$ and tunable bulk and surface anisotropics yield additional complexity. This makes the magnetism of the particles more involved.

On example is the single- or multidomain character of the magnetic ground state. Kittel's critical single-domain radius $R_{\mathrm{SD}}$ describes the transition from a single-domain state to a two-domain configuration. ${ }^{1}$ However, Kittel's expression is limited to spherical particles, ignores hysteresis effects, and breaks down completely for domain-wall thicknesses larger than the dot size. Furthermore, the critical singledomain radius is an equilibrium property, obtained by comparing energy minima. By contrast, hysteresis is a nonequilibrium phenomenon involving energy barriers, so that the single- or multidomain character of a particle is irrelevant to hysteresis. ${ }^{4,5}$ The popular but incorrect equating of singledomain magnetism and coherent rotation, as epitomized by the term elongated single-domain particle, has its origin in the exclusive focus on soft and semihard magnets a few decades ago. In fact, the onset of nonuniform (incoherent) reversal in perfect ellipsoids of revolution is governed by the exchange length. This quantity is anisotropy-independent and, in hard magnets, much smaller than $R_{\mathrm{SD}}$. Figure 1 illustrates this point for a defect-free spherical particle. a) Author to whom correspondence should be addressed; electronic mail:
rskomski@neb.rr.com
This article deals with generally nonellipsoidal nanodots of thickness $t$, width $W$, and length $L \geqslant W$. Analytical calculations and numerical simulations are used to investigate the ground state and the hysteresis of the particles.

\section{MICROMAGNETIC BACKGROUND}

Both equilibrium and nonequilibrium magnetic properties depend on geometrical parameters, such as dot size, and on micromagnetic parameters, such as the anisotropy $K_{1}$. Dimensional analysis of the micromagnetic free energy $4,6,7$ yields two fundamental lengths, namely the wall-width parameter $\delta_{0}=\left(A / K_{1}\right)^{1 / 2}$ and the exchange length $l_{\mathrm{ex}}$ $=\left(A / \mu_{0} M_{s}^{2}\right)^{1 / 2}$. The wall-width parameter determines the thickness of the domain wall ${ }^{8}$ separating magnetic domains of different magnetization directions and describes the spatial response of the magnetization due to local perturbations. It varies from about $1 \mathrm{~nm}$ in extremely hard materials to several $100 \mathrm{~nm}$ in very soft materials. The exchange length is the length below which atomic exchange interactions dominate typical magnetostatic fields and determines, for example, the transition from coherent rotation to curling. Since typical ferromagnets have magnetizations of the order of $1 \mathrm{~T}$

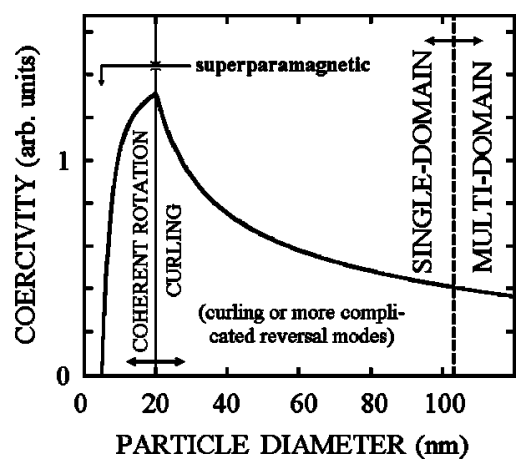

FIG. 1. Phase diagram for small hard magnetic spheres. Since the magnetocrystalline anisotropy is much larger then the magnetostatic self energy, there is a broad region where the particle is single domain but reverses by incoherent rotation. 


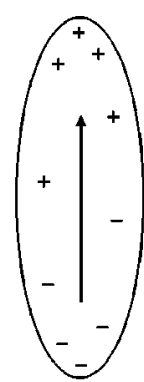

(a)

(b)

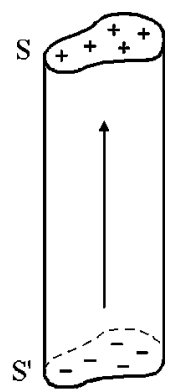

FIG. 2. Surface poles at particles with parallel faces.

and exchange stiffnesses of the order of $10 \mathrm{pJ} / \mathrm{m}, l_{\text {ex }}$ is between 1 and $2 \mathrm{~nm}$ for a broad range of materials. ${ }^{5,9}$

The magnetostatic self-interaction term favors magnetic domains with partial or complete flux closure which are separated by comparatively narrow domain walls. ${ }^{1,2,7,8,10}$ The self-interaction energy competes against the domain-wall energy, and domain formation in spheres is favorable when the radius is larger than the critical single-domain radius $R_{\mathrm{SD}}$ $=36 \sqrt{A K_{1}} / \mu_{0} M_{s}^{2} .{ }^{1}$ In very hard magnets, $R_{\mathrm{SD}}$ exceeds 1 $\mu \mathrm{m}$. However, the critical single-domain size of nonsperical structures is strongly geometry dependent.

\section{RESULTS AND DISCUSSION}

The determination of the critical single-domain size requires the knowledge of the magnetostatic self interaction, as epitomized by the demagnetizing factor $D$. General ellipsoids have been investigated by Osborn, ${ }^{11}$ but there are no closed expressions for arbitrary principal axes and for nonellipsoidal shapes. When the magnetization is uniform and parallel to a principal anisotropy axis, the demagnetizing factor of homogeneously magnetized bodies $E_{\mathrm{MS}}$ $=D_{\|} \mu_{0} M_{s}^{2} V / 2$. Here $V$ is the volume of the magnet

$$
E_{\mathrm{MS}}=\mu_{0} M_{s}^{2} / 8 \pi \iint \sigma(r) \sigma\left(\mathbf{r}^{\prime}\right)\left|\mathbf{r}^{\prime}-\mathbf{r}\right|^{-1} d S d S^{\prime}
$$

is the magnetostatic self-interaction energy, and $\sigma=\mathbf{M} \cdot \mathbf{n}$ is the magnetic surface charge. Figure 2 shows the surface charges for (a) an ellipsoid of revolution and (b) for an particle with parallel top and bottom surfaces but arbitrary cross section. In (b), the charges are much more localized, which enhances the self energy of Eq. (1) and facilitates domain formation. In lowest order

$$
D_{\|}=1 / 2 \pi L S_{0} \int_{\text {top }} \int_{\text {top }}\left|\mathbf{r}^{\prime}-\mathbf{r}\right|^{-1} d S d S^{\prime}-\frac{S_{0}}{2 \pi L^{2}},
$$

where $S_{0}$ is the cross-section area (top surface area) and $L$ is the length of the particle. The double integral describes the self interaction at the particle's top, and the $1 / L^{2}$ term is the interaction between the poles. For a square cross section $t^{2}$, Eq. (2) yields

$$
D_{\|}=\frac{c_{0} t}{L}-\frac{t^{2}}{2 \pi L^{2}},
$$

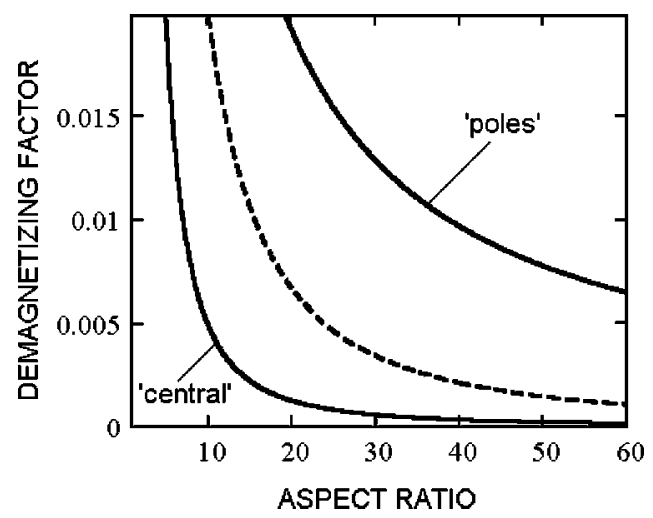

FIG. 3. Demagnetizing factors for square-rod particles. The "poles" approximation is exact for large aspect ratios. The dashed line is the prediction for ellipsoidal particles.

where $c_{0}=0.506$ is obtained by numerical integration of Eq. (2). This differs significantly from the results for elongated ellipsoids of revolution, where $D_{\|} \sim t^{2} / L^{2}$ plus a logarithmic correction. This is because most of the surface charge of long ellipsoids is on the sides, which reduces the self interaction. Figure 3 compares the present approximation with the demagnetizing factor deduced from the demagnetizing field in the center of the particle in Fig. 2(b). Figure 3 means that the central-field and ellipsoidal approximations significantly underestimate the trend towards domain formation.

In a strict sense, domain theory is limited to magnets whose size is larger than the domain-wall width $\delta_{B}$. Otherwise, inhomogeneous magnetization states extend throughout the particle and $\nabla M \sim M_{s} / R$. The competing magnetostatic and exchange energies then scale as $\mu_{0} D M_{s}^{2} R^{3}$ and $A R$, respectively, and a strongly nonuniform ground state is
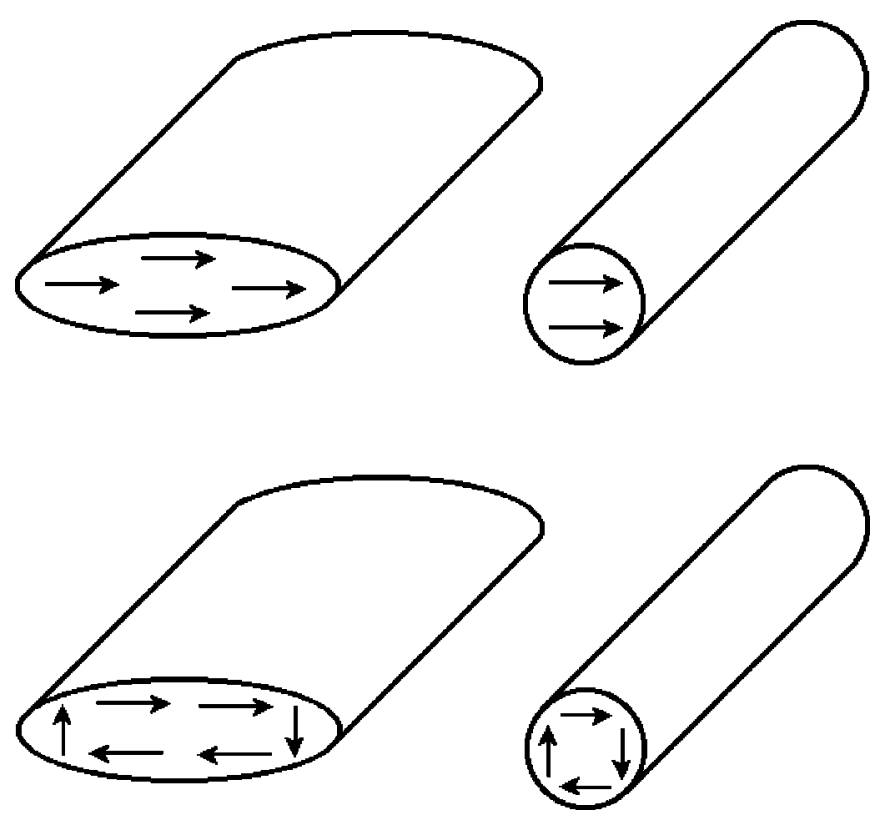

FIG. 4. Magnetization reversal in long nanodots with ellipsoidal cross sections: coherent rotation (top row) and curling (bottom row). The external magnetic field and the main magnetization direction are parallel to the long axes of the ellipsoids; the arrows show small perpendicular perturbations of the magnetization. The field is applied parallel to the long axis of the particles. 


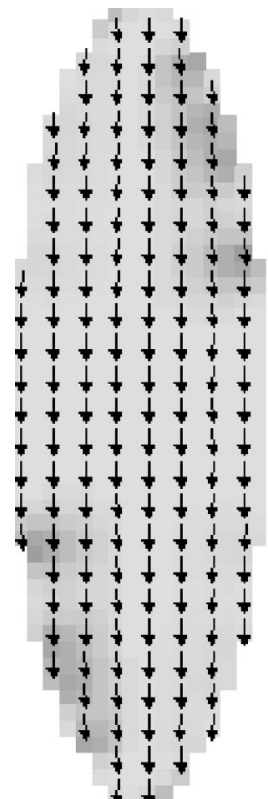

(a)

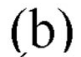

FIG. 5. Spin structure of in-plane nanodots, as obtained from the micromagnetic calculations: (a) single-domain and (b) flux-closure states.

realized when $R$ is much larger than $\sqrt{A /\left(\mu_{0} D M_{s}^{2}\right)}$ $=D^{-1 / 2} l_{\text {ex }}$. Here $D$ refers to the shape anisotropy of the particles, which is, in thin-film dots, proportional to the ratio of film thickness to dot diameter. It can be shown that this scaling law is valid for $\mu_{0} D M_{s}^{2} \gg K_{1}$. In the opposite limit, $\mu_{0} D M_{s}^{2} \ll K_{1}$, the critical radius scales as $R_{\mathrm{SD}} / D$, where $R_{\mathrm{SD}}$ is Kittel's expression.

The problem of magnetization reversal is quite different from the problem of ground-state domains. The involved mathematics is fairly complicated, ${ }^{6,7}$ but the basic physics is seen from the following scaling argument. For ellipsoids of revolution, the exchange energy density $A(\nabla M)^{2} / M_{s}^{2}$ scales as $A / R^{2}$, where $R$ is the radius of the ellipsoid. This energy competes against the gain in magnetostatic energy, whose density is on the order of $\mu_{0} M_{s}^{2}$. As a consequence, curling is favorable when the radius is larger than some multiple of the exchange length: $R_{\mathrm{coh}}=c_{\mathrm{coh}} l_{\mathrm{ex}}$. In practice, $l_{\mathrm{ex}} \approx 2 \mathrm{~nm}$ and $c_{\mathrm{coh}} \approx 5$, so that $R_{\mathrm{coh}} \approx 10 \mathrm{~nm}$ for a wide range of materials. ${ }^{4,5}$

This is no longer true for flat bodies, such as those shown in Fig. 4, where it is necessary to distinguish between thickness $t$ and width $W$. The exchange and magnetostatic energy densities scale as $A / t^{2}$ and $t \mu_{0} M_{s}^{2} / W$, where $t / W$ is the corresponding demagnetizing factor. As a consequence, the critical thickness for curling, $t_{\mathrm{coh}}$, scales as $l_{\mathrm{ex}}\left(W / l_{\mathrm{ex}}\right)^{1 / 3}$. This means that flattening an ellipsoid tends to suppress curling. A crude estimate is $t_{\text {coh }}=200 \mathrm{~nm}$ for $W=10 \mu \mathrm{m}$, and below this thickness, coherent rotation is more favorable than curling.

Since the above considerations are only semiquantitative, we have used micromagnetic simulations to study the spin structure and the hysteresis loops of thin-film nanodots. The thickness of the films, $t=40 \mathrm{~nm}$, is easily accessible by

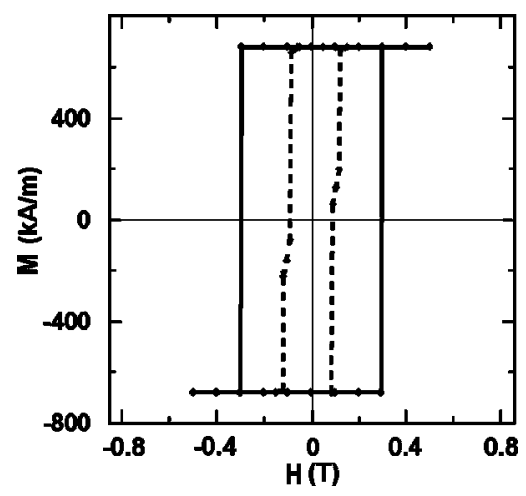

FIG. 6. Hysteresis loops for ellipsoidal permalloy nanodots of dimensions $50 \times 400 \times 40 \mathrm{~nm}^{3}$ (solid line) and $60 \times 90 \times 40 \mathrm{~nm}^{3}$ (dashed line).

methods such as focused ion-beam milling. The calculations were performed using an OOMMF NIST code, with a cell size of $2 \mathrm{~nm}$. Figures 5 and 6 show simulated spin structures and hysteresis loops for the permalloy dots, respectively. Dots having a length of $400 \mathrm{~nm}$ and a width of $50 \mathrm{~nm}$ are single domains in the ground state and exhibit rectangular hysteresis loops. By contrast, dots having a length of $90 \mathrm{~nm}$ and a width of $60 \mathrm{~nm}$ exhibit a flux-closure domain and a slightly nonrectangular hysteresis loop.

\section{CONCLUSIONS}

In summary, the ground-state domain structure and the magnetization reversal of thin-film nanodots are investigated by model calculations and micromagnetic simulations. At the lower end of the size and thickness range, there are no domains in the dots. With the exception of very flat and very elongated dots, large particles are multidomain with nearly complete flux closure and complex reversal behavior. In general, magnetostatic self interactions in real elongated particles, such as square rods, are much stronger than those in ellipsoidal particles, because in the latter case the surface charges are quite delocalized. This enhances the trend towards domain formation. Intermediate dot sizes lead to flux closure in low fields and to small but well-defined coercivities. This may be useful for future applications where logic operations are realized by magnetic bits.

\section{ACKNOWLEDGMENTS}

This work is supported by ARO, NSF-MRSEC, the W. M. Keck Foundation, and CMRA.

${ }^{1}$ C. Kittel, Rev. Mod. Phys. 21, 541 (1949).

${ }^{2}$ R. M. Bozorth, Ferromagnetism (Van Nostrand, Princeton, N.J., 1951).

${ }^{3}$ M. Zheng et al., Appl. Phys. Lett. 79, 2606 (2001).

${ }^{4}$ R. Skomski, J. Phys.: Condens. Matter 15, R841 (2003).

${ }^{5}$ R. Skomski and J. M. D. Coey, Permanent Magnetism (Institute of Physics, Bristol, 1999).

${ }^{6}$ W. F. Brown, Micromagnetics (Wiley, New York, 1963).

${ }^{7}$ A. Aharoni, Introduction to the Theory of Ferromagnetism (Oxford University Press, Oxford, 1996).

${ }^{8}$ F. Bloch, Z. Phys. 74, 295 (1932).

${ }^{9}$ R. Skomski, H.-P. Oepen, and J. Kirschner, Phys. Rev. B 58, 3223 (1998).

${ }^{10}$ A. Hubert and R. Schäfer, Magnetic Domains (Springer, Berlin, 1998).

${ }^{11}$ J. A. Osborn, Phys. Rev. 67, 351 (1945). 